\title{
Determinación de factores de adhesión asociados a la formación de biopelícula en aislamientos clínicos de Staphylococcus aureus y Staphylococcus epidermidis
}

\author{
Determination of adhesion factors associated with biofilm formation in \\ clinical isolates of Staphylococcus aureus and Staphylococcus epidermidis
}

Gladys Pinilla ${ }^{1}$, Angela Bautista ${ }^{2}$, Claudia Cruz ${ }^{2}$, Bibiana Chavarro ${ }^{1}$, Jeannette Navarrete ${ }^{1}$, Liliana Muñoz ${ }^{1}$, Jennifer Gutiérrez ${ }^{1}$

\section{Resumen}

Los factores de adhesión son determinantes de virulencia que se expresan en microorganismos que tienen la capacidad de formar biopelícula, contribuyendo a la gravedad de infecciones intrahospitalarias. Dentro de estos componentes de la superficie microbiana que reconocen moléculas de adhesión de matriz conocidas como MSCRAMMs, se incluyen el factor de unión a fibronectina A y B, (FnbA y B) factor de aglutinación A y B (ClfA y B) y factor de unión a fibrinógeno (Fib), que se han descrito en Staphylococcus aureus y reaccionan con proteínas de la matriz extracelular humana. El objetivo de este estudio fue determinar la presencia de estos factores de adhesión relacionados con la formación de biopelicula en Staphylococcus. Método. Se caracterizaron fenotípica y genotípicamente 30 aislamientos clínicos de Staphylococcus aureus y Staphylococcus epidermidis, provenientes de pacientes inmunocomprometidos en tres instituciones hospitalarias de Bogotá. La producción de Biopelícula se determinó mediante Rojo Congo y Cristal violeta y mediante PCR convencional y múltiplex se amplificaron los genes $F n b A$ y $B, C l f A$ y $B$ y Fib, así como los genes del operón ica $A D B C$. Resultados. Todos los aislamientos clínicos fueron positivos genotípica y fenotípicamente para la producción de Biopelícula, encontrándose la presencia del operón completo en el $88.9 \%$, los factores $C l f A$ y $C l f B$ en un $70 \%$; Fib en un $60 \%, F n b B$ en un $23 \%$ y FnbA en el 17\%. Conclusiónes. En este estudio se evidenció la presencia de estos factores de virulencia en $S$. epidermidis, los cuáles hasta el momento se han reportado únicamente en S.aureus. Este hallazgo es importante ya que se sugiere la relación con transferencia horizontal de genes entre estas especies, siendo el S. epidermidis un importante reservorio genético, y un importante patobionte causal de infecciones nosocomiales, asociado con dispositivos médicos.

Palabras claves: Biopelícula, factores de adhesión, Staphylococcus aureus, Staphylococcus epidermidis.

1. Facultad de Ciencias de la Salud, Programa de Bacteriología y Laboratorio clínico, Universidad Colegio Mayor de Cundinamarca

2. Semillero Grupo REMA Universidad Colegio Mayor de Cundinamarca. 


\begin{abstract}
Adhesion factors are virulence determinants that are expressed in microorganisms with the ability to form biofilms, contributing to the severity of nosocomial infections. Among these microbial surface components recognizing adhesive matrix molecules (MSCRAMMs), are fibronectin binding $A$ and $B$ (fnbA and B) clumping $A$ and B (ClfA and B) and fibrinogen binding (Fib) factors. All of these have been described in Staphylococcus aureus and react with human extracellular matrix proteins. The goal of this study was to determine adhesion factors related to the biofilm formation in Staphylococcus. Method. For these purpose, 30 clinical isolates of Staphylococcus aureus and Staphylococcus epidermidis, from immunocompromised patients in three hospitals in Bogotá were characterized both, genotypically and phenotypically. The biofilm formation was determined through Congo Red and Violet Crystal and the genes FnbA, B, ClfA, B, Fib and operon ica ADBC were amplified through conventional and multiplex PCR. Results. Every clinical isolate were genotypically and phenotypically positive for the biofilm formation, being found the presence of the whole ica ADBC operon in 88,9\%. The ClfA and ClfB were found by $70 \%$; Fib 60\%, fnbB $23 \%$ and $17 \%$ of $f n b A$. Conclusions. This study proved the presence of these virulence factors in S. epidermidis, which so far have only been reported in S. aureus. This finding is important because it suggestes the relationship with horizontal gene transfer between these species, being the $S$. epidermidis an important genetic reservoir and a causal patobiont of nosocomial infections associated with medical devices.
\end{abstract}

Keywords: Biofilm, adhesion factors, Staphylococcus aureus, Staphylococcus epidermidis.

\section{Introducción}

Los Staphylococcus spp involucrados en infecciones intrahospitalarias han desarrollado varios mecanismos de resistencia antimicrobiana, como la formación de biopelícula mediada por el operón icaA$D B C$, contribuyendo a la gravedad de la infección, por disminución en la absorción del antibiótico; $y$ por ende, la persistencia del patógeno $(1,2)$.

Los patógenos nosocomiales involucrados en este tipo de infecciones son principalmente Staphylococcus aureus y Staphylococcus epidermidis que se comportan como patógenos oportunistas, catalogados según el microbioma humano como patobiontes en personas inmunocomprometidas y que presentan un gran potencial de formar biopelícula, ya que se encuentra en piel y en superficies mucosas, los cuales pueden ser introducidos a los tejidos durante la implantación de dispositivos médicos $(3,4)$.

Dentro de los actores principales en el proceso de formación de biopelícula, se han descrito una serie de moléculas denominadas factores de adhesión, que son moléculas de tipo proteico que expresa la bacteria para facilitar su adhesión a una superficie, siendo este el primer y determinante paso en el establecimiento de una película bacteriana (4).

Comúnmente estos factores de adhesión o proteínas son referenciadas como moléculas de la 
matriz de adherencia o MSCRAMMs (por sus siglas en inglés: Microbial Surface Components Recognizing Adhesive Matrix Molecules), de las que se conoce que tienen la capacidad de unirse a la matriz extracelular de proteínas del plasma del huésped tales como: fibrinógeno, fibronectina, colágeno, vitronectina y laminina (4-6) . Estudios realizados en $S$. aureus han descrito una serie de genes que codifican para diferentes MSCRAMMs; los genes $C l f A, C l f B$ y fib que codifican para proteínas de unión al fibrinógeno y las proteínas de unión a la fibronectina, codificadas por los genes $f n b A$ y $f n b B$, proteínas denominadas de igual forma que sus genes codificantes (7-9).

Específicamente el factor Clumping o de aglutinación A, codificado por el gen $C l f A$, fue el primer MSCRAMM identificado, es el más conservado entre especies y facilita la unión del patógeno a el C-terminal de la cadena gamma del fibrinógeno presente en el plasma sanguíneo del huésped (6). A su vez este ha demostrado promover la evasión bacteriana de la defensa del huésped, en parte por la inhibición del proceso de fagocitosis realizado por neutrófilos y macrófagos a través de mecanismos dependientes e independientes al fibrinógeno (7). El factor Clumping o de aglutinación B $(C l f B)$ es una adhesina bifuncional que se expresa únicamente en la superficie celular durante la fase exponencial de crecimiento y es capaz de unirse a las cadenas alfa del fibrinógeno, así como a la citoqueratina diez (CK10), una proteína estructural de las células epiteliales escamosas, por lo que ha sido implicado en la colonización del S. aureus en el epitelio nasal $(10,11)$.

Las proteínas de unión a la fibronectina (FnbA) y (FnbB) se han identificado en los géneros Staphylococcus y Streptococcus (12). Son proteínas codificadas por genes estrechamente vinculados, ya que poseen una alta homología en su secuencia. Estas trabajan juntas para el establecimiento de la infección y son indispensables en la adhesión inicial del $S$. aureus al epitelio. La FnbA es indispensable en la internalización de las bacterias a la célula huésped mientras que la FnbB estimula la fagocitosis (13).

Estas proteínas tienen la capacidad de unirse al fibrinógeno y a la elastina gracias al dominio N-terminal que poseen (14); seguido por una región que contiene 11 repeticiones de aminoácidos en el caso de la FnbA y 10 repeticiones para la FnbB que son las responsables de que se dé la unión con la fibronectina, permitiendo así la invasión de células endoteliales por parte del Staphylococcus aureus (15-16).

Dado que estos factores de adhesión (ClfA, ClfB, FnbA, FnbB y fib) hasta el momento han sido reportados solo en $S$. aureus, en este estudio se pretende evidenciar si se encuentran presentes también en $S$ epidermidis. Este hallazgo sería importante ya que se sabe poco acerca de cómo $S$. epidermidis interactúa con proteínas de la matriz, siendo este el agente causal de infección en mayor frecuencia en pacientes neonatales e inmunocomprometidos, y por lo tanto, un potente patobionte.

\section{Metodología}

\section{Aislamientos bacterianos}

Se analizaron 30 cepas de Staphylococcus spp provenientes de pacientes de tres instituciones hospitalarias de Tercer Nivel de Bogotá, que presentaron infecciones asociadas con implantes de dispositivos médicos. Estos aislamientos fueron caracterizados fenotípica y genotípicamente para confirmación de especie y se determinó la capacidad para formar biopelícula, mediante agar rojo congo y cristal violeta, para verificar la adherencia bacteriana valorada semicuantitativamente por la técnica propuesta por Freeman y colaboradores $(17,18)$.

\section{Extracción de ADN}

La extracción de ADN se llevó a cabo mediante pre-tratamiento con lisozima $2,5 \mathrm{mg} / \mathrm{ml}$ y lisostafina $1,2 \mathrm{mg} / \mathrm{ml}$ a una temperatura de $37^{\circ} \mathrm{C}$ por 
60 minutos y se siguió el protocolo para bacterias Gram positivas de Wizard ${ }^{\circledR}$ Genomic DNA Purification Kit (Promega, Winsconsin USA)

\section{Determinación de la presencia de los genes del} operon ica y de factores de adbesión

La detección de los genes que componen el operón ica $A D B C$ e ica $R$, encargado de mediar la formación de biopelícula en $S$. epidermidis y $S$. aureus, se realizó mediante la técnica de PCR múltiplex, usando las siguientes condiciones: $\mathrm{MgCl} 2$ 2,5mM; dNTPs $200 \mu \mathrm{M} ; 0,25 \mu \mathrm{M}$ de cada primer y Taq polimerasa $1 \mathrm{U}$ (invitrogen, $\mathrm{Ca}-$ lifornia, USA). Las condiciones de amplificación fueron 30 ciclos: denaturación a $94^{\circ} \mathrm{C}$ por 15 segundos, hibridación a $55^{\circ} \mathrm{C}$ por 30 segundos y elongación por 1 minuto a $72^{\circ} \mathrm{C}$. Se utilizaron las cepas USA 300 y la ATCC 12228 como controles positivo y negativo, respectivamente.

Para la amplificación de adhesinas (clfA, clfB, fib, $f n b A$ y $f n b B$ ) se realizó PCR múltiplex, utilizando los cebadores descritos por Tristan et al (19), con las siguientes condiciones: $\mathrm{MgCl} 22 \mathrm{mM}$; dNTPs $200 \mu \mathrm{M}$; cebadores $0,5 \mu \mathrm{M}$ de cada gen y Taq polimerasa 1,25U (invitrogen, California, USA), durante 25 ciclos: desnaturalización por $1 \mathrm{minu}-$ to, hibridación a $55^{\circ} \mathrm{C}$ por 1 minuto, extensión a $72^{\circ} \mathrm{C}$ por 1 minuto. Se utilizaron las cepas USA 300 y la ATCC 12228 como controles positivo y negativo, respectivamente.

\section{Resultados}

\section{Aislamientos clínicos}

Se estableció la relación entre la especie de Staphylococcus y el tipo de muestra obtenida por cada entidad participante en el estudio encontrando que la frecuencia de aislamientos fue para $S$. epidermidis $(80 \%)$ y para $S$. aureus (20\%). En las 3 instituciones hospitalarias el mayor porcentaje de aislamientos tanto de $S$. epidermidis como $S$. aureus fue proveniente de muestras de hemocultivos, seguido de puntas de catéter. Adicionalmente, se encontró que la mayoría de los aislamientos clínicos en este estudio provenían de pacientes en estado de inmunosupresión (datos no mostrados).

\section{Determinación de las MSCRAMMs}

Se amplificaron fragmentos de los genes que codifican factores de adhesión $c l f A, c l f B, f i b, f n b B, y$ $f n b A$ mediante PCR multiplex (Figuras 1 y 2); los genes clfA, clfB fueron los mayormente encontrados $(70 \%)$, seguidos de fib (60\%), fnbB (23\%) y fnbA (17\%).

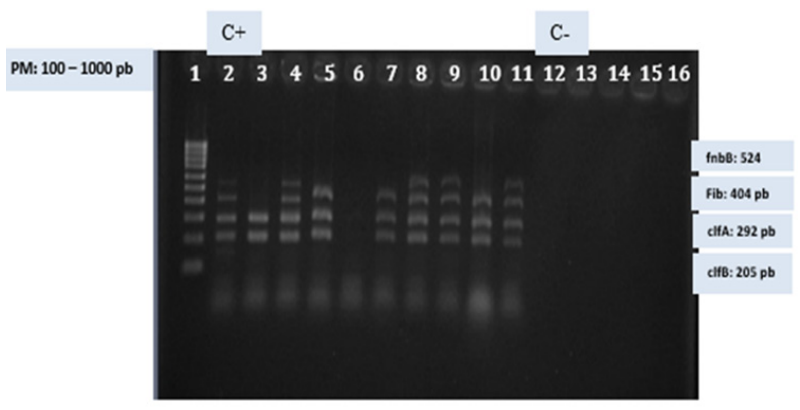

Figura 1. Productos de Amplificación de los factores de adhesión clfA, clfB, fib, y fnbB mediante PCR multiplex: Carriles. 1. marcador de peso molecular, 2.control positivo para $c l f A$, $c l f B, f i b, y$ fnbB cepa USA $300 \mathrm{~S}$. aureus, 3 al 11. Aislamientos clínicos positivos para los factores ClfA (292pb), ClfB (205 pb), FnbB (524pb) y fib (404 pb).

\section{Factores de adbesión y tipo de muestra}

Se estableció la correlación entre la presencia de cada factor vs. la especie de Staphylococcus y sitio de infección, y se encontró que los genes que codifican para los factores de adhesión analizados en este estudio y hallados en $S$. aureus fueron todos provenientes de hemocultivos y no se encontró el gen $f n b B$ en ninguna de las muestras analizadas. Para $S$. epidermidis todos los factores se encontraron en mayor proporción en muestras de hemocultivo y punta de catéter; sin embargo, no se hallaron los factores en la muestra de absceso analizada. Los factores más frecuentes en todas las muestras fueron $c l f A, c l f B$ y $f i b$, Figura 2. 


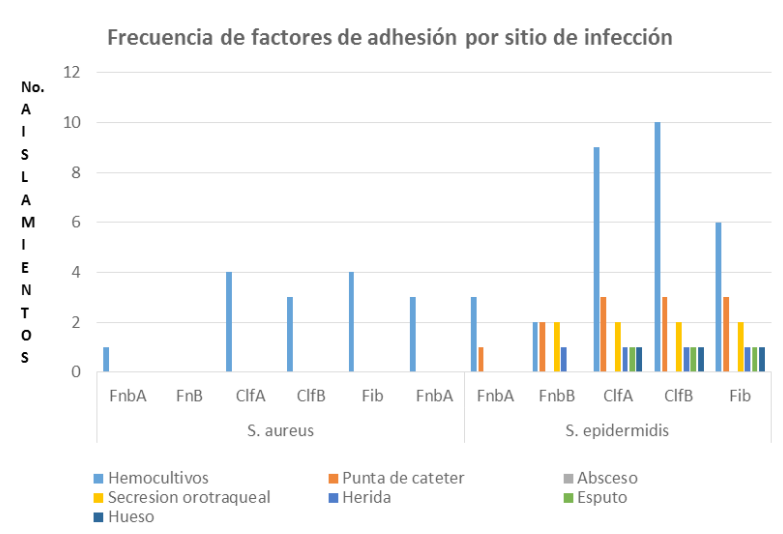

Figura 2. Frecuencia de cada factor de adhesión según el tipo de Staphylococcus en cada uno de los tipos de muestra analizados.

Caracterización fenotípica y genotípica de la biopelícula

Los aislamientos clínicos estudiados, presentaron las pruebas cualitativa de rojo congo, y semicuantitativa de cristal violeta positivas y la frecuencia de aparición de los genes del operón ica $A D B C$ e ica $R$ fue la siguiente: presencia de los 5 genes, es decir, del operón completo, $88.9 \%$; cuatro de los genes (ica $R$, ica $D B C$ ), 7,4\% y dos de los genes (ica $R$, icaD), 3,7\%

\section{Discusión}

En el presente estudio se evidenció que componentes de la superficie microbiana, que reconocen moléculas de adhesión a la matriz extracelular, conocidas como MSCRAMMs, considerados factores de virulencia en Staphylococcus, específicamente descritas para $S$. aureus, se encuentran presentes también en aislamientos clínicos de $S$. epidermidis. Este hallazgo es importante ya que este último microorganismo, considerado comensal de piel, o autobionte, es en la actualidad un importante reservorio genético, y a su vez, debido a la transferencia horizontal de genes entre estas especies, ha pasado a ser considerado potencial patobionte, dentro de la clasificación del Microbioma humano. Adicionalmente, proporciona información acerca de cómo S. epidermidis podría interactúar con proteínas de la matriz extracelular humana y su relación con la formación de biopelícula, siendo este un importante factor de persistencia bacteriana en infecciones frecuentes en pacientes neonatales e inmunocomprometidos (20). No obstante a pesar de que existe mucha información sobre MSCRAMMs en Staphylocuccus, se sabe poco sobre los mecanismos moleculares por los cuales estas proteínas conducen a la adhesión bacteriana (21).

Las adhesinas $C l f A, f n b A$ y $f n b B$ se unen a los residuos $C$ - terminal de la cadena $Y$ del fibrinógeno, la cual es una región que está involucrada en la agregación plaquetaria; en Staphylococcus epidermidis la adhesina propia de éste, la $s d r G$ se une a la cadena $\beta$ del fibrinógeno, el cual es un sitio de clivaje para la trombina, lo que hace que estos Staphylococcus al inmovilizar el fibrinógeno, estén involucrados en el desarrollo de aterosclerosis, trombosis, CID, y por tanto, en el riesgo de enfermedad cardiovascular (22).

Significativamente todos los residuos de $C l f B$ que se unen a la citoqueratina 10 (K10) y a la cadena a del fibrinógeno son péptidos conservados en Staphylococcus aureus, representando 48 variantes únicas de la proteína. Es así como $A s n-238$ y $T h r-$ 383 interactúan con el fibrinógeno pero no con la citoqueratina, mientras que la Gly-521 hace contacto únicamente con esta (23).

En este estudio los genes codificantes de las adhesinas ClfA, ClfB y fib se encontraron en los mismos tipos de muestras: hemocultivos, puntas de catéter, secreciones oro-traqueales y herida de miembro inferior derecho y hueso. Este hallazgo podría estar relacionado con la similitud estructural existente entre $C l f A$ y $f i b$, ya que se ha demostrado que ambas proteínas en el dominio $A$ $\mathrm{N}$-terminal poseen tres subdominios N1, N2 y N3; los aminoácidos del subdominio N1 de ClfA y fib tienen el 19\% de identidad, mientras que los subdominios N2 y N3 comparten alrededor del $60 \%$ de identidad (24).

Las MSCRAMMs FnbA y FnbB promueven la acumulación de la biopelícula en cepas de Sta- 
phylococcus aureus con resistencia a la meticilina (MRSA). Es así como cepas MRSA pueden expresar proteínas de superficie tales como Bap, Sas $G$, Sas $C$, FnbA y $F n b B$ que promueven la acumulación en la formación de biopelícula de manera independiente del operón icaADBC (25).

La $F n b A$ y la $F n b B$ median la acumulación bacteriana requiriendo $\mathrm{Zn}{ }_{2}$. Se ha encontrado que al remover el $\mathrm{Zn}+{ }_{2}$ utilizando DTPA como quelante, se inhibe la acumulación bacteriana, pero no afecta la expresión o la integridad de las proteínas lo que implica que ambas proteínas promueven la formación de biofilm por un mecanismo dependiente del $\mathrm{Zn}+{ }^{2}$ (26).

Los resultados obtenidos en el presente trabajo muestran que $F n b A$ fue la MSCRAMMs con menor frecuencia, con un $17 \%$ del total de los aislamientos, hallándose únicamente en hemocultivos y en punta de catéter. FnbB solo se halló en aislamientos de $S$. epidermidis a partir de hemocultivos, puntas de catéter, secreción orotraqueal y esputo. No obstante, dada la importancia que tienen estos factores en la formación de biopelículas bacterianas, es necesario determinar no solo la presencia de los genes que codifican para estos factores sino también la expresión genética de estos.

En cuanto a la prevalencia de las MSCRAMMs en Staphylococcus relacionados con sepsis, se reporta que ClfA está presente en el $100 \%$ de los aislamientos, $C l f B$ entre el 98 y $100 \%$, fib está presente en un 90 a $100 \%$; mientras que el $F n b B$ sí varia en Staphylococcus sensibles a meticilina (MSSA), donde se encuentra en el 30\% de los aislamientos mientras que en Staphylococcus resistentes (MRSA) se reporta entre 53.3 a $50 \%$ y para el $F n b A$ se encuentra entre el 100 y el 63.6\% (24).

En la presente investigación se encontró $C l f A$ y $C l f B$ en un $70 \%$, Fib en un $60 \%, F n b B$ en un $23 \%$ y $F n b A$ en el $17 \%$ de los aislamientos clínicos, evidenciando una alta frecuencia de estas adhesinas en pacientes inmunosuprimidos. Staphylococcus tienen la capacidad de ser internalizados por diversos tipos de células como: células endoteliales, células epiteliales, fibroblastos, osteoblastos, queratinocitos, además de los fagocitos comunes. Es importante destacar que la expresión de estas MSCRAMMs no siempre sigue un patrón, aunque algunas sean más conservadas que otras (24).

S. epidermidis es un microorganismo comensal humano lo que hace de esta bacteria un soporte y reservorio óptimo para la transferencia de genes de resistencia. Por ejemplo, se habla que S. epidermidis posee elementos genéticos móviles nombrados como casetes cromosómicos (SCCmec) que hacen que aumente la resistencia a la meticilina, por lo que ha sugerido que estos casetes fueron transferidos de S. epidermidis a $S$. aureus (27).

La transferencia genética en las biopelículas de $S$. aureus se da por medio de plásmidos a través de conjugación y movilización, lo que determina la resistencia a antibióticos y la transferencia de adhesinas (MSCRAMMs) para la formación de la biopelícula. Así mismo se sabe que casi todos los patógenos bacterianos formadores de biopelícula contienen mecanismos de transferencia génica horizontal inducibles $(28,29,30,31)$; en este contexto en nuestro estudio se plantea que pueden ser éstos los responsables de la transferencia de las MSMCRAMS entre $S$. aureus y epidermidis, efectuada mediante la combinación de elementos genéticos móviles. Lo anterior se plantea con base en Investigaciones anteriores, en las cuales evidenciamos en los aislamientos clínicos estudiados en el presente trabajo, la presencia de plásmidos con la capacidad de transportar integrones que confieren mecanismos de resistencia a las bacterias, mediante la inclusión de casetes genéticos, lo que posiblemente explicaría el encontrar estos genes que inicialmente solo se reportaron para $S$. aureus, también en $S$. epidermidis, hallazgo soportado por el mecanismo de transferencia horizontal de genes $(32,33)$. 
Se sugiere que las cepas de relevancia clínica contienen plásmidos conjugativos que facilitan la formación de la biopelícula; un ejemplo de ello es Escherichia coli, que codifica el plásmido $\mathrm{F}$ que actúa como un factor de adhesión en la superficie celular actuando en las interacciones de célula a célula para la conformación de biopelículas (34). No obstante es importante mencionar que muchos organismos que no pueden transportar plásmidos producen solo microcolonias pero no biopelícula, pero cuando estos organismos reciben plásmidos de bacterias donantes, estos empiezan a producir biopelículas (35-37).

Por otra parte, varios autores relacionan que las cepas que poseen el operón icaADBC forman necesariamente biopelícula, mientras que otros, sostienen que la presencia del operón no es suficiente sino que son necesarios otros factores como las autolisinas, MSCRAMMs, etc.; Sin embargo y dada la importancia de este cluster genético en relación al papel que cumple en la formación de biopelícula, Pinilla y colaboradores identificaron la presencia del operón ica $A D B C$ en el $88 \%$ de los aislamientos clinicos y el $90 \%$ de estas poseían el regulador negativo icaR, lo cual llevó al diseño de péptidos análogos a $i c a R$ con especificidad antibiopelícula, como posible alternativa terapéutica $(28,31,32)$.

Las MSCRAMMs no se encontraron en todos los aislamientos a pesar de que estos fueron formadores de biopelículas determinada tanto fenotípica, como genotípicamente. Esto podría explicarse por las combinaciones individuales entre MSCRAMMs, que influencian sucesos microbianos de adhesión o pueden generar la no expresión completa de los mismos; además de que se pueden presentar variaciones alélicas entre adhesinas del mismo tipo que pueden aumentar o reducir su unión a las células humanas (24).

Por otra parte, se destaca que en las tres instituciones hospitalarias, se evidenció una alta frecuencia de MSCRAMMs en hemocultivos. Las
MSCRAMMs que han sido más asociadas a la infección en el torrente sanguíneo son ClfA y FnbA, produciendo así mismo fuertes biopelículas. Staphylococcus aureus y Staphylococcus epidermidis son responsables de más de la mitad de todas las infecciones nosocomiales sanguíneas en Estados Unidos (38).

Staphylococcus epidermidis reporta una alta resistencia bacteriana en hemocultivos reportándose una mortalidad entre el 18\% a 57\%. En biopelículas que se forman en implantes óseos, el 80\% de ellas están siendo causadas por especies de $S$. aureus y S. epidermidis (39).

En cuanto a las estrategias más prometedoras para el tratamiento de la biopelícula se encuentran el diseño y uso de vacunas, la terapia génica, alternativas de prevención de formación de biopelícula evitando la adherencia y la proliferación bacteriana, lo que permite el control de las células en fase planctónica, por medio de los tratamientos de la superficie del implante o dispositivo médico (40-45).

Finalmente, el estudio de las MSCRAMMs es de suma importancia ya que no solo permiten la unión a las proteínas de la matriz extracelular, sino que permiten la acumulación y proliferación bacteriana, convirtiéndolas en uno de los factores de virulencia más importantes promovidos por la biopelícula y de esta forma, constituirse en un blanco terapéutico importante para controlar su formación y por ende disminuir la virulencia del patógeno.

\section{Agradecimientos}

Este proyecto fue financiado por la Universidad Colegio Mayor de Cundinamarca y COLCIENCIAS Convocatoria 657-2014 Código COL0041221, Contrato RC651-2014. 


\section{Referencias}

1. Betancourth M, Botero J, Patricia S. Biopelículas: una comunidad microscópica en desarrollo. Colombia médica 2004; 35(1):34-39.

2. Henríquez D, Leal A. Resultados del proyecto: "Impacto clínico y económico de la resistencia bacteriana en hospitales del distrito". Boletín informativo grupo GREBO 2007: Disponible en http://grebo.org/grebo_site/jgrebo/documentos/Boletin\%20Informativo\%20No\%20S1\%202010.pdf

3. Herrera M. El papel del biofilm en el proceso infeccioso y la resistencia. Nova 2004; 2:71-80.

4. Costerton J, Stewart P, Greenberg E. Bacterial Biofilms: a common cause of persistent infection. Science 1999; 284:13181322 .

5. Granger J. La placa dental como biofilm ¡cómo eliminarla? RCOE 2005; 10: 431-439.

6. Heukelekian H, Heller A. Relation between food concentration and surface for bacterial growth. J Bacteriol 1940; 40:4 547-558.

7. Costerton J, Geesey G and Cheng J. How Bacteria Stick. Scientific American 1978; 238 86-95

8. Arciola C, Campoccia D, Ravaioli S, Montanaro L. Polysaccharide intercellular adhesin in biofilm: structural and regulatory aspects. Front Cell Infect Microbiol. 2015; 5:7

9. Heilmann C, Schweitzer O, Gerke C, et al. Molecular basis of intercellular adhesion in the biofilm-forming Staphylococcus epidermidis. Mol Microbiol. 1996; 20: 1083-1091.

10. Walsh EJ, Miajlovic H, Gorkun OV, Foster TJ. Identification of the Staphylococcus aureus MSCRAMM clumping factor B (ClfB) binding site in the alpha C-domain of human fibrinogen. Microbiology 2008; 154:550-8.

11. Ganesh VK, Barbu EM, Deivanayagam CC, et al. Structural and biochemical characterization of Staphylococcus aureus clumping factor B/ligand interactions. J Biol Chem 2011; 286:25963-72.

12. Vengadesan K, Sthanam N. Structural biology of Gram- positive bacterial adhesins. Protein Sci. 2011; 20: 759-772.

13. Dowd S, Sun Y, Secor P, et al. Survey of bacterial diversity in chronic wounds using pyrosequencing, DGGE, and full ribosome shotgun sequencing. BMC Microbiol 2008; 8 (1):43.

14. Stoodley P, Sauser K, Davies D.G, and Costerton W. Biofilms as complex differentiated communities. Annu Rev Microbiol. 2002; 56: 187-209.

15. Flemming $\mathrm{H}, \mathrm{Neu} \mathrm{T}$ and Wozniak D. The EPS matrix: the "house of biofilm cells". J Bacteriol. 2007; 189: 7945-47.

16. Rice K, Mann E, Endres J, et al. The cidA murein hydrolase regulator contributes to DNA release and biofilm development in Staphylococcus aureus. Proc Natl Acad Sci USA 2007; 104:8113-18.
17. Freeman DJ, Falkiner FR, Keane CT. New method for detecting slime production by coagulase negative staphylococci. J Clin Pathol. 1989 Aug;42(8):872-4.

18. Christensen GD, Simpson W a., Younger JJ, Baddour LM, Barrett FF, Melton DM, et al. Adherence of coagulase-negative staphylococci to plastic tissue culture plates: A quantitative model for the adherence of staphylococci to medical devices. J Clin Microbiol. 1985;22(6):996-1006.).

19. Tristan A, Ying L, Bes M, Etienne J, Vandenesch F, Lina G . Use of multiplex PCR to identify Staphylococcus aureus adhesins involved in human hematogenous infections. J Clin Microbiol, 2003. 41(9): p. 4465-7.

20. Méric G, Miragaia M, de Been M, et al. Ecological Overlap and Horizontal Gene Transfer in Staphylococcus aureus and Staphylococcus epidermidis. Genome Biol Evol. 2015; 7(5):13131328.

21. Herman-Bausier P, Dufrêne YF. Atomic force microscopy reveals a dual collagen-binding activity for the staphylococcal surface protein SdrF. Mol Microbiol. 2016 Feb;99(3):611-21. doi: $10.1111 / \mathrm{mmi} .13254$

22. Christensen GD, Bisno AL, Simpson WA, Beachey EH. Adherence of slime-producing strains of Staphylococcus epidermidis to. Infect Immun, 1982: 37: 318-26.

23. Vernachio JH, Bayer AS, Ames B, et al. Human Immunoglobulin G Recognizing Fibrinogen-Binding Surface Proteins Is Protective against both Staphylococcus aureus and Staphylococcus epidermidis Infections In Vivo. Antimicrob Agents Chemother. 2006; 50(2):511-518

24. O’Connell D.P., Nanavathy,T., McDevitt,D., Gurusiddappa,S., Höök,M. and Foster,T.J. The fibrinogen-binding MSCRAMM (clumping factor) of Staphylococcus aureus has a Ca2+-dependent inhibitory site. J. Biol. Chem 1998; 273: 6821-6829.

25. Geoghegan JA, Monk IR, O'Gara JP, Foster TJ. Subdomains N2N3 of Fibronectin Binding Protein A Mediate Staphylococcus aureus Biofilm Formation and Adherence to Fibrinogen Using Distinct Mechanisms. Journal of Bacteriology. 2013; 195(11):2675-2683.

26. O’Neill E, Pozzi C, Houston P, et al. A Novel Staphylococcus aureus Biofilm Phenotype Mediated by the Fibronectin-Binding Proteins, FnBPA and FnBPB J Bacteriol. 2008;190(11):38353850 .

27. Campoccia D, Montanaro L, Arciola C R. The significance of infection related to orthopedic devices and issues of antibiotic resistance. Biomaterials 2006; 27 (11): 2331-9.

28. Pinilla, G., Muñoz, L., Navarrete, J., Castro, B., Chavarro, B., Avila, L. Transferencia de genes de resistencia en aislamientos clínicos de Staphylococcus spp: Integrones Clase I en ADN plasmídico y cromosomal. En: Memorias: Congreso Latinoamericano de Microbiología (XXI ALAM); Santos Brasil; 2012. p.66-67. 
29. Pinilla, G., Muñoz, L., Navarrete, J., Salazar L Diseño de Péptidos con actividad analoga al represor IcaR de Staphylococcus spp. formador de biopelícula, Revista Colombiana de Química. Universidad Nacional. Vol 44 No. 22015 pp. 5-9 El DOI asignado para este artículo es http://dx.doi.org/10.15446/rev. colomb.quim.v44n2.55213

30. Muñoz, L., Salazar, LM:, Botero S., Navarrete J., Pinilla G. Possible antibiofilm effect of peptides derived IcaR repressor of Staphylococcus epidermidis Responsible for Hospital acquires sepsis. Advances in Computacional Biology. 2014, 232: 91-95

31. Pinilla, G., Muñoz, L., Navarrete, J., Chavarro, B., Salazar L. Molecular Characterization of Clinical Isolates of Staphylococcus spp. For Design of antibiofilm peptides as a possible alternative therapy. 2014 abstract 114th American Society of Microbiology en Boston Massachusetts. Disponible en: http:// app.coreapps.com/tristar_asm2014/abstract/ff4e29439dd7a$7331 \mathrm{dd} 90 \mathrm{c} 3 \mathrm{c} 98 \mathrm{dff} 692$

32. Rojas J, Urrutia J. Formación de Biopelícula en Staphylococcus $s p p$ : caracterización fenotípica y genotípica [Tesis de pregrado]. Universidad Colegio Mayor de Cundinamarca. 2014

33. Otto M. Staphylococcus epidermidis - the "accidental" pathogen. Nat Rev Microbiol. 2009; 7(8):555-567

34. Büttner H, Mack D, Rohde H. Structural basis of Staphylococcus epidermidis biofilm formation: mechanisms and molecular interactions. Front Cell Infect Microbiol. 2015; 5:14

35. Chavarro B, Pinilla G, Castro B, Yomayusa N, Moreno JE, Escobar JA, Vanegas N. Determinación de la presencia y transcripción de factores de virulencia en aislamientos colombianos de Staphylococcus aureus resistente a meticilina adquirido en la comunidad. Infectio. 2010;14(S1)80.

36. Méric G, Miragaia M, de Been M, et al. Ecological Overlap and Horizontal Gene Transfer in Staphylococcus aureus and Staphylococcus epidermidis. Genome Biol Evol. 2015; 7(5):13131328.

37. McCrea W, Hartford Orla, et al. The serine- aspartate (Sdr) protein family in Staphylococcus epidermidis. Microbiology. 2000; 146: 1535-1546.

38. Davis S.L., Gurusiddappa,S., McCrea,K.W., Perkins,S. and Höök,M. SdrG, a fibrinogen-binding bacterial adhesin of the MSCRAMM subfamily of the Staphylococcus epidermis, targets the thrombin cleavage site in the $\beta$ chain. J. Biol. Chem.2001, 276: 27799-27805.

39. Savage VJ, Chopra I, O’Neill AJ. Staphylococcus aureus Biofilms Promote Horizontal Transfer of Antibiotic Resistance. Antimicrob Agents Chemother. 2013; 57(4):1968-1970.

40. Pontes C, Alves M, Santos C, Ribeiro MH, Gonçalves L, Bettencourt AF, Ribeiro IA Can Sophorolipids prevent biofilm formation on silicone catheter tubes? Int J Pharm. 2016; 513 (1-2):697-708.
41. Pedroso SH, Sandes SH, Luiz KC, Dias RS, Filho RA, Serufo JC, Farias LM, Carvalho MA, Bomfim MR, Santos SG. Biofilm and toxin profile: A phenotypic and genotypic characterization of coagulase-negative staphylococci isolated from human bloodstream infections. Microb Pathog. 2016;100:312-318. doi: 10.1016/j.micpath.2016.10.005.

42. Desroche N, Dropet C, Janod P, Guzzo J. Antibacterial properties and reduction of MRSA biofilm with a dressing combining polyabsorbent fibres and a silver matrix. J Wound Care. 2016; 25(10):577-584.

43. Galvez, Z. Y. A. and V. E. M. Burbano. Bacillus: género bacteriano que demuestra ser un importante solubilizador de fosfato. NOVA Publicación en Ciencias Biomédicas. 2015; 12(22): 165-177.

44. Corrales, L. C., et al. Bacterias anaerobias: procesos que realizan y contribuyen a la sostenibilidad de la vida en el planeta. 2015; Nova 13(24): 55-82.

45. Rodríguez, O. E., Andrade, W. A., Díaz, F. E., \& Moncada, B. Actividad antimicrobiana de líquenes de la cuenca alta del rio Bogotá. 2015; Nova, 13(23). 